\title{
A CASE OF EMPYEMA,
}

\author{
WITH \\ PULMONARY GANGRENE FOLLOWING ENTERIC \\ FEVER, TREA'TED BY PERFLATION.
}

BY

WILLIAM EWART, M.D.CANTAB., F.R.C.P., AND

R. FITZROY BENHAM, M.R.C.S.

WITH REMARKS ON THE METHOD OF PERFLATION, AND ITS VALUE IN THE TREATMENT OF EMPYEMA.

By WILLIAM EWART, M.D.

Received August 23rd, 1886-Read March 8th, 1887.

[THE early notes of the case are by Mr. Benham, who attended the patient throughout his illness.]

"On April 24th, 1886, I first saw Charles C-,

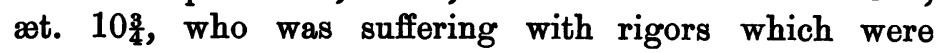
followed by fever; and I was informed that prior to my visit he had been feeling unwell for about two or three weeks. The family history was healthy, and there had been no previous illness except scarlet fever in early childhood and chorea minor a few months prior to the present illness, for which I attended the patient. I soon discovered, by the special features of the temperature chart, of the evacuations, and of the cutaneous spots, that 
this was a case of enteric fever. Astringents were prescribed to check the diarrhœa, and the pyrexia was partly controlled by antipyrin, quinine and acid having been tried in vain. The violent delirium, which lasted continuously for sixteen days, also abated soon after antipyrin was administered. Even during the delirium localised tenderness could be detected over the right iliac fossa. By the end of the fourth week the patient had so far improved as to be able to play with his toys. A few days later, however, an attack of general peritonitis set in which was marked by the usual symptoms. The urine deposited lithates, but was otherwise normal. The diet at that time was still restricted to fluids.

"Five days after the onset of the peritonitis, which had meanwhile greatly diminished in severity, pain was felt on deep breath, and on cough at the upper part of the left parasternal region. The pain gradually increased, and on the third day some dulness on percussion at the left base was recognised, together with exaggerated breathing at the seat of pain. The patient rapidly lost ground, and after about eight days from the onset of the chest-trouble he was suffering from severe exhaustion and dyspnœa on the slightest attempt at movement. He lay on his left side breathing rapidly (at about 40 per minute), emaciated, suffused, with parched red tongue, and mouth extensively studded with aphthæ. The cough was feeble and hacking, and he had a hectic temperature. The left chest, which I found the previous day to measure one inch more than the right, was absolutely dull from the second rib downwards, the dulness extending to the right for one and a half inches beyond the manubrium sterni. No heart-beat could be detected on the left side, but the cardiac impulse was plainly visible in the right chest, and was felt and heard with greatest intensity at a spot one and a quarter inches to the right of the right nipple in the fifth intercostal space. The left chest was motionless and bulged in its upper part. Loud blowing breathing and coarse râles were audible above the left 
second rib, and less abundantly over the upper part of the right lung anteriorly. Loud blowing breathing was also heard to the right of the manubrium. The voicesounds were not anywhere tubular in character. Feeling that paracentesis should be performed without delay I consulted Dr. Ewart on June 11th."

The physical signs described in Mr. Benham's report having been confirmed, and the patient's pulse being of fair strength, we determined to aspirate the empyema immediately. General anæsthesia was induced and the needle was inserted into the fifth intercostal space, close to the anterior axillary line. After escape of three quarters of a pint only of a shreddy pus, not fœtid, but possessing a tenacious, stale " animal" odour, the flow ceased, and could not be re-established, although the cannula was carefully probed and found patent. The heart receded only a little way towards the left, but the dyspnœe was considerably lessened. We determined to open the chest next day, and on June 12th two free openings were made (under the carbolized spray from a Benham spray-producer), one at the site of the previous aspiration, the other dorsally in the ninth interspace, in vertical line with the angle of the left scapula. The pus resembled closely that obtained by aspiration; its amount was estimated at about three pints. A probe introduced at the anterior orifice could be moved freely to a depth of four or five inches, and could be pushed upwards and inwards as far as a spot corresponding to the level of the second right costo-sternal junction. Avoidance of all fluid injections having been agreed upon as a principle of treatment, a continuous drainage-tube was introduced and the wounds were dressed with a thin bag of carbolized gauze loosely filled with wood-wool impregnated with corrosive sublimate, and covered with the usual mackintosh. This formed not only a most efficient absorbent pad but also completely sealed the wounds. Meanwhile the patient was much relieved. His breathing, which had been very rapid, became quiet and he was able to lie on his right side. Owing to the free

vol. LXx. 
oozing of discharge it was necessary to renew the dressings the same evening.

On the 13th breathing was audible over the upper part of the lung, especially at the back; the discharge was moderate in amount. On the 14th the discharge was rather foul but not abundant; two short tubes with flanges were substituted for the single long tube. On the 15th (being the fourth day from the date of the operation), perflation of the chest with carbolized air was performed with the help of a Richardson's bellows, and of a washbottle containing 10 per cent. solution of carbolic acid in water. Care was taken that the air, delivered into the chest through the anterior wound by an india-rubber tube, should not escape anteriorly, but issue through the posterior orifice.

A few seconds after the beginning of perflation the posterior opening, which in the recumbent posture of the patient was the most dependent part of the chest, became blocked, and its lips bulged outwards, causing slight pain. In another moment, under the influence of rising pressure, a solid substance was forced through the aperture; this was followed by a large mass of shreddy false membrane and by upwards of one ounce of fœid pus. The pain immediately subsided, as well as the slight congh which had been set up at first. ${ }^{1}$ Ventilation was kept up for fifteen minutes, but nothing further was discharged. The carbolized spray had played on the wounds throughout and the same kind of dressing was reapplied. (A few days later, finding that the skin had become irritable and scattered with red spots, Mr. Benham dusted the surfaces with a powder consisting of oxide of zinc and boracic acid.) The temperature, which for the last three days had returned to normal degrees, did not rise, and the patient spent a comfortable night. The solid lump was found on inspection to be a wedge of whitish-grey, solidified and necrosed pulmonary substance, which measured 2 centimetres by $1 \cdot 4$ and by 0.9 , in its longest diameters. On June 16th was

1 Cough was not observed at subsequent dressings in this case. 
painlessly expelled, by the same method, a fresh supply of false membrane, with a little pus ; and again on the 17th a small shreddy mass, weighing about four drachms, together with two fluid drachms of pus. An examination of the chest, made on the 15th, had revealed the presence of loud bronchial breathing opposite the left first and second cartilages; this was replaced, on the 17 th, by vesicular breathing. The vesicular murmur was audible, before the dressings were detached from the wounds, as low as the fourth rib anteriorly, and as the serenth rib behind. After their removal the breathing became less vesicular and mixed with amphoric notes. At the base, front and back, the breathing was purely amphoric. The heart had not returned to its normal apex-site, although it closely approached that spot. A probe inserted anteriorly still reached with facility the region covered by the second left costo-sternal junction, and could be sent far and free towards the posterior opening; but in the axillary direction the mobile surface of the lung could be felt at the level of the third rib. Owing to the narrowness and obliquity of the intercostal spaces the posterior part of the pleura could not be explored satisfactorily.

On the 18th, 19th, and 20th the dressings showed only stains of pus ; and hardly any discharge was expelled by perflation. On the 22nd (being the tenth day from the date of operation), about one ounce of slightly turbid, blood-stained serum escaped; and about six drachms of the same fluid, but of clearer quality, were discharged on the 24th. On this date auscultation yielded, before any air was admitted, vesicular sounds posteriorly as far as the base of the lung and anteriorly close up to the wound, but not in the interval between the two wounds. Air having been let in, the vesicular breath-sound was transformed into a harsh blowing, which in the scapular region was accompanied with a faint amphoric sound; whilst true amphoric breathing extended over an area one and a half inches in diameter between the wounds. No abnormality of the voice-sounds was found except a little way above the 
posterior wound, where they possessed a bleating character. When the probe was introduced it came into contact with the lung within a distance of three inches in the upward direction.

It was inferred from the results of this day's examination that the lung was almost fully expanded during the periods of air-tight closure between successive dressings, leaving only a small cavity at the base of the pleura, and that it was partly adherent to the chest wall in the posterior mid-axillary region.

On the 28th the fluid exudation was about the same in amount; the probe could still be sent into a large free space. On July 4th, being anxious to close the anterior wound, and finding that the posterior opening was becoming uncomfortably small, we agreed that the latter should be enlarged, and this was done under ether. When the finger was introduced through the enlarged wound it impinged upon the tough surface of the lung, and below this it was possible to pass a probe in the direction of the anterior opening. On this occasion, in order to avoid the escape into the chest of any of the blood from the incision (which bled rather freely) the wound was kept exposed for a considerable time, and for upwards of three quarters of an hour, from first to last, perflation was applied under spray. On July 5th not more than one drachm of serum was secreted ; on the 6th, 7th and 9 th, only a few drops. The anterior tube was removed on the 10th, and a smaller tube was inserted behind. On the 12th, exactly one month from the date of thoracentesis, the posterior tube was found lying out of the wound ; the next day only a pin-hole opening remained, and on July 15th the wound was healed. Breathing was now everywhere audible, and was of good quality except at the axillary base. The left shoulder was drooped from nervousness, but there was hardly any permanent deformity. The right chest measured $12 \frac{5}{8}$ inches at the level of the nipple; the left $12 \frac{3}{8}$ inches.

Remarks on the case.-Without being pathologically remarkable, the case is of some interest, inasmuch as it 
presents an unbroken chain of pathological events. That the bowel was extensively ulcerated may be safely assumed from the severity of the pyrexia, and of the typhoid symptoms. The enteric lesions were probably responsible directly for the peritonitis, and indirectly, by way of embolism, for the pulmonary gangrene; and the latter was followed by suppuration within the pleura. In favour of the view that the gangrene was the outcome of thrombosis or embolism strong support is derived from the appearances of the specimen expelled from the pleura. Its pulmonary nature is readily identified by the smooth glistening surface covering two of its sides and by the faint pigmentary tracings of the lobules, whilst its shape shows it to belong to the pulmonary fringe. The line of separation is indicated on one of the smooth surfaces by a rim of yellow inspissated pus closely parallel to the ulcerated edge. On one of the two surfaces roughened by ulceration is seen a small cylindrical pit resembling the bed of an absent vessel. This, it is assumed, was the site of the original pulmonary lesion. (Attention may be drawn incidentally to the fact that the specimen was evacuated through an external opening of apparently smaller diameter than would allow of its easy passage.) The shreddy false membranes were yellowish-grey, and consisted of fibrin mixed with pus; only one of the smaller pieces possessed a red tinge such as would identify it with the blood-clot. The presence within the pleura of material such as we have described would in itself lend a feature of gravity to an empyema irrespective of any constitutional exhaustion preceding, as in this case, the chest affection. That in a case thus complicated suppuration should have ceased as early as the tenth day, and made place for a serous flow, is a result perhaps not exceptional, but worthy of record.

This result is, in our opinion, to be ascribed to the successful evacuation of the cavity within the first week; and the early expansion of the compressed lung was in great measure due to the care bestowed on the dressings with a 
view to air-tight closure of the wounded side. It must be confessed that the final operation for enlarging the posterior opening was undertaken unnecessarily under the impression that the expansion of the lung and the obliteration of the cavity were not keeping pace with the healing of the external wounds. The auscultatory evidence of the extensive re-establishment of breathing during the intervals of closure of the wounds should have been more implicitly trusted. Had this evidence been acted upon the empyema might perhaps have been permanently closed within fourteen days after the paracentesis. With an actual duration of four weeks the case may nevertheless be looked upon as successful in point of time.

\section{Remarks on the Method of Perflation and on its value in the Treatment of Empyema.}

(Before proceeding with these remarks it is my pleasant duty to express my indebtedness to $\mathrm{Mr}$. Benham, not only for having agreed to the application of a novel method, but for his valuable assistance in the endeavour to render it a success, and for practical suggestions in the manipulation of the apparatus. To his able surgical co-operation is due in a large measure the successful result obtained in the case.)

Hippocrates, although free from the indiscriminating dread of air which arose after his time (his only fear was lest the lung should become parched, a danger which he sought to obviate by injecting oil and wine), does not appear to have utilized air surgically in the treatment of empyema. Modern English works contain no mention of the injection of air as a means of treatment in this affection. But in a pamphlet by Audouard ('De l'Empyème,' Paris, 1828), the following suggestive remark appears as a mere parenthesis : "Et pourquoi ne se servirait on pas, de l'air comme moyen médicamenteux ?" 
This suggestion remained unnoticed, and the prevailing prejudice against air held continued sway. In 1841, in an inaugeral thesis on empyema, which was supposed to reflect recent knowledge and practice, Sédillot laid down the following proposition (p. 173) : "Une indication complexe, très importante pour la cure, est celle de laisser toujours dans la poitrine assez de pus pour éviter l'introduction de l'air et trop peu pour écarter les parois thoraciques." The deep-rooted objection to the escape of air into the chest had its origin in the observation that pus in contact with air underwent putrefaction. Cases, however, were not wanting in which putrescence occurred even after its exclusion, and Sédillot was driven to use the lame argument that air did harm by its bulk, mechanically preventing the expansion of the lung.'

Within recent times the lingering distrust of air suggested to Bowditch the aspiration method, a valuable addition to surgical resources; and the success of this method may have served for a time to emphasize an erroneous belief. But since the beginning of the Listerian era we have learnt to regard air with less diffidence, and the treatment of empyema may now be expressed in a formula of surprising simplicity : "a timely and sufficient incision to be kept aseptic and open." In this form it was applied by Dr. Markham Skerritt, in $1876,{ }^{2}$ without the complication of any fluid injection.

Air was capable, however, of something better than harmless neutrality. It was pressed into the service by Roser and subsequently by Quincke. To Roser ${ }^{3}$ I must resign the claim of priority for a method which, up to three days before writing these lines I had held to be not only original but novel. Roser's method differs from my own less in its principle than in some of its details. The air injected is

1 The discussion on thoracentesis before the Académie de Médecine in 1872 is of historical interest in connection with the alleged dangers from the admission of air into the pleura.

2 ' Brit. Med. Journal,' July 22nd, 1876.

3 'Archiv der Heilkunde,' v, p. 8t, 1864. 
nnwashed, an ordinary air-syringe is used, and a second chest-tube is provided for the outflow. Roser's views and his practice entirely agree with my own with regard to the mechanical uses of the injected air, but from his short description it does not appear to what extent he employed perflation systematically as a healing agent. Roser's results, as far as reported, were highly satisfactory, even in the presence of such complications as pulmonary or bronchial fistula. It is surprising that in spite of this success the method should have failed to become generally known, or fairly tested.

H. Quincke ${ }^{1}$ suggested as an improvement an ingenious apparatus, whereby air and fluid were alternately injected. The leading pretext for this innovation was the alleged danger arising from shifting the patient's position during the first days after incision. Quincke's method, which can be applied strictly in situ, is excellent theoretically, but its results do not sufficiently commend it.

Perflation, as I have practised it, consists in systematic " through-ventilation" of the suppurating cavity. I have selected the term perflation as the shortest and most explicit word conveying that the chest is not inflated, nor merely injected with air, but "blown through." The method has been described in the 'Lancet' (July 31st, 1886, p. 226), and need only be briefly sketched here. The following apparatus is necessary : (1) a Richardson's bellows; (2) india-rubber tubing to conduct the air from the bellows into the bottle and from the bottle into the chest; lastly, (3) a tall wash-bottle containing a short column of strongly carbolised water $\left(\frac{1}{10}\right)$, to act as a purifier and as a valve for the air to be forced through it. This bottle, in winter, should stand in warm water.

In cases where a single opening has been made into the chest, if the tube be introduced to a sufficient depth, air conveyed by the tube from the bellows will pervade the whole space before it escapes at the orifice by the side of the tube; and if any obstruction should exist near the 1 'Berliner klin. Wochenschrift,' 1872, p. 65. 
orifice the air would be delivered into the cavity beyond. Where two openings have been made, one of them will admit the tube and will require to be closed air-tightly during perflation, the other remaining patent. In either case obstruction may arise from some internal plug as soon as the patient has been so placed that the wound of exit is absolutely dependent. If air be now injected, its tendency will be to drive out the plug.

The following mechanical principles are at the root of the method : (a) air diffuses into every recess of a given space, whereas fluids gravitate; (b) a current of air penetrating with a certain force into an air-containing cavity will distribute some of this force in every direction; (c) owing to the specific lightness of air, fluids or solids will not be suspended by it, but will inevitably fall in it to the most dependent spot.

It is freely admitted that fluid injections can also be made to enter into universal contact with a cavity, and that they also are capable of transmitting even pressures. With these objects in view, various authorities ${ }^{1}$ have utilized the principle of the syphon. But with all fluid injections it remains a matter of chance whether the solid to be expelled will gravitate through the fluid medium to the exact spot desired. Moreover, several objections must be raised against the method of filling, emptying, and refilling the chest by means of a syphon. Two, three, or more pints of fluid make up a weight which can hardly be tolerated by the pleura with comfort nor, if hydraulic pressure be superadded, with safety. The lung is thereby submitted to sustained compression of a very inelastic kind ; and further, in virtue of its great buoyancy it is not unlikely to be floated up, out of position, thus straining its vessels.

But the chief practical defect common to the syphon method, and to all methods involving fluid injections, is the difficulty of absolutely emptying the pleura by their

1 F. Cresswell Hewett, ‘ Brit. Med. Journal,' March 11, 1876 ; Kashimura of Tokio, 'Berliner klinische Wochenschrift,' No. 3, 1880. 
means, and the uncertainty in which the operator is left on this score. Solid material may remain behind as well as fluid. To speak of fluids only, injections of any kind, if allowed to stagnate in the pleura, cannot fail to set up or perpetuate irritation.

From the patient's point of view, perflation is mechanically preferable to fluid injections. It is simple, painless, free from shudders and drenchings, and performed with a minimum of disturbance, and, as far as my experience goes, with practical freedom from danger.

Judged by its results in the case narrated above, perflation seems to possess the following advantages as a method of treatment for empyema :

(1) It facilitates the early removal from the chest of putrid residues or of the recent products of inflammation.

(2) In the same measure it tends to shorten the period of suppuration; and

(3) To lessen the danger of permanent shrinking of the lung; and, therefore, also

(4) To lessen the necessity for resection of ribs.

(5) It leaves the chest relatively dry, and practically free from any foreign material, since air is rapidly absorbed by the pleura.

This paper would not be complete without some allusion to the question of the surgical value of local ventilation. Some authorities appear to have seen good results from this form of treatment. Bouisson ${ }^{1}$ submitted superficial wounds to the direct draught of a bellows for frequent periods of fifteen minutes. A scab usually formed, under which reparative processes advanced more rapidly than under moist dressings or ointments. The same method appears to have been successfully tried by Sir James Simpson. Dr. Ogilvie Wills saw wounds treated in this manner unite without one drop of pus. Dr. Krönlein ${ }^{3}$ devotes an important work to the study of the treatment

1 'Brit. and Foreign Medico-Chirurg. Review,' i, 1859, p. 546.

2 'Lancet,' i, 1873, p. 907.

3 'Die offne W'unJbehandlung,' Zürich, 1872. 
of wounds without any applications impeding local ventilation, and appends to his book a useful bibliography.

Roser (loc. cit.) observed in cases treated by his method a very favorable influence on the abscess walls. In his own words, "their shrinking proceeded so rapidly that one might assume that the stimulus of air had a special action in that direction." My own case gives countenance to the same assumption.

It may be questioned whether in single empyemas, or in the healing stage of empyemas originally complicated with gangrene or false membrane, the pleural surface presents any true granulations. More probably granulations are to be found only in cases of inveterate suppurations of the pleura with fistulous opening. If the injection of simple or of medicated air should prove to possess a tendency to change the latter condition into the former, to convert a pus-secreting into a serum-secreting surface, or if in the absence of any inherent virtue attaching to air the general conditions of the treatment could be shown to favour such a result, then the therapeutical value of the method which I advocate would be established.

Perflation, in this communication, has been considered exclusively in connection with empyema. Its applicability to other pulmonary conditions and to other regions of the body will suggest itself without need of any remarks from me. The mode and duration of its application might be varied to any extent, as well as the antiseptics used in its performance. These are matters for experimental surgery. For my part, even should the usefulness of perflation fall short of larger expectations, I shall feel content if in many cases of empyema it may, in the future, be found as beneficial as in the cases we have related.

Dr. Delépine's Report on the small mass removed from the pleural cavity: The microscopical examination shows that the specimen is a small mass of lung tissue. The pleura and subjacent alveoli, with some carbon pigmentation, can be easily recognised, and the alveoli are distended 
with granular débris, probably the remains (decolourised) of some extravasated blood.

(For report of the discussion on this paper, see 'Proceedings of the Royal Medical and Chirurgical Society,' New Series, vol. ii, p. 221.) 